\title{
THE INDEX COMPLEX OF A MAXIMAL SUBALGEBRA OF A LIE ALGEBRA
}

\author{
DAVID A. TOWERS
}

\author{
Department of Mathematics and Statistics \\ Lancaster University \\ Lancaster LA1 4YF \\ England \\ d.towers@lancaster.ac.uk
}

\begin{abstract}
Let $M$ be a maximal subalgebra of the Lie algebra $L$. A subalgebra $C$ of $L$ is said to be a completion for $M$ if $C$ is not contained in $M$ but every proper subalgebra of $C$ that is an ideal of $L$ is contained in $M$. The set $I(M)$ of all completions of $M$ is called the index complex of $M$ in $L$. We use this concept to investigate the influence of the maximal subalgebras on the structure of a Lie algebra, in particular finding new characterisations of solvable and supersolvable Lie algebras.

Mathematics Subject Classification 2000: 17B05, 17B20, 17B30, 17 B50.

Key Words and Phrases: Lie algebras, maximal subalgebra, index complex, ideal index, solvable, supersolvable, Frattini ideal.
\end{abstract}

\section{Introduction}

Let $M$ be a maximal subalgebra of the Lie algebra $L$. A subalgebra $C$ of $L$ is said to be a completion for $M$ if $C$ is not contained in $M$ but every proper subalgebra of $C$ that is an ideal of of $L$ is contained in $M$. The set 
$I(M)$ of all completions of $M$ is called the index complex of $M$ in $L$. This is analogous to the concept of the index complex of a maximal subgroup of a finite group as introduced by Deskins in [6]; this concept has since been further studied by a number of authors, including Ballester-Bolinches and Ezquerro ([2]), Beidleman and Spencer ([5], Deskins ([7]), Mukherjee ([8]), and Mukherjee and Bhattacharya ([9]). The objective of this paper is to investigate relationships between the properties of maximal subalgebras of $L$ and the structure of $L$ itself corresponding to similar relationships in the group-theoretic case.

It is easy to see that the sum of all ideals of $L$ that are proper subalgebras of $C$ is itself a proper subalgebra of $L$. We define the strict core (resp. core) of a subalgebra $B \neq 0$ to be the sum of all ideals of $L$ that are proper subalgebras (resp. subalgebras) of $B$, and denote it by $k(B)$ or $k_{L}(B)$ (resp. $\left.B_{L}\right)$. Notice that the strict core can differ from the core even when $B$ is an ideal of $L$ : for example, if $B$ is a one-dimensional ideal of $L$. The subalgebra $C$ is then a completion of the maximal subalgebra $M$ of $L$ (that is, $C \in I(M))$ if $L=\langle M, C>$ and $k(C) \subseteq M$. Completions always exist, as the following lemma establishes.

Lemma 1.1 If $M$ is a maximal subalgebra of $L$ then $I(M)$ is non-empty; in fact, $I(M)$ contains an ideal of $L$.

Proof. Clearly the set of ideals of $L$ which do not lie in $M$ is a non-empty partially ordered set; choose $C$ to be a minimal element of this set. Then $L=M+C=<M, C>$ and $k(C) \subseteq M$, so $C \in I(M)$.

In section two we study completions that are ideals and show that if $C$ and $D$ are two such completions of the same maximal subalgebra $M$ then $C / k(C) \cong D / k(D)$. This allows us to define the ideal index of $M$ in $L$. Next we characterise solvable and supersolvable Lie algebras in terms of the ideal index of their maximal subalgebras. We then consider completions $C$ that are ideals and for which $C / k(C)$ is abelian. A characterisation of solvable Lie algebras and of the solvable radical is given in terms of such completions.

In section three maximal completions are studied. It is shown that over an algebraically closed field a Lie algebra is solvable if and only if every maximal subalgebra of $L$ has an abelian maximal completion. This is analogous to a result of Deskins (see [7]) for groups. The Lie algebraic result, however, is false if the underlying field is not algebraically closed, even if it has characteristic zero. 
The final section is devoted to subideal completions. A characterisation of supersolvable Lie algebras in terms of such completions is found that is analogous to a group-theoretic result of Ballester-Bolinches and Ezquerro (see [2]). The Lie algebraic proof, however, is completely different, as the group theoretic result relies on properties that do not hold in the case of Lie algebras.

Throughout, $L$ will denote a finite-dimensional Lie algebra over a field $F$. If $A$ and $B$ are subalgebras of $L$ for which $L=A+B$ and $A \cap B=0$ we will write $L=A \oplus B$. The ideals $L^{(k)}$ of the derived series are defined inductively by $L^{(0)}=L, L^{(k+1)}=\left[L^{(k)}, L^{(k)}\right]$ for $k \geq 0$; we also write $L^{2}$ for $L^{(1)}$. If $A$ is a subalgebra of $L$, the centralizer of $A$ in $L$ is $C_{L}(A)=\{x \in L:[x, A]=0\}$.

\section{Ideal completions and the ideal index}

If $C$ is an ideal of $L$ and $C \in I(M)$ we call $C$ an ideal completion of $L$. In this case $C / k(C)$ is a chief factor of $L$ which is avoided by $M$. Up to isomorphism it is uniquely determined by $M$.

Theorem 2.1 Let $C$ and $D$ be ideal completions of the maximal subalgebra $M$ of $L$. Then $C / k(C) \cong D / k(D)$.

Proof. Let $L$ be a Lie algebra of minimal dimension having a maximal subalgebra $M$ with two ideal completions $C$ and $D$ such that $C / k(C) \not$ $D / k(D)$. If $k(C) \cap k(D) \neq 0$ then by factoring out $k(C) \cap k(D)$ and using the minimality of $L$ we see that $C / k(C) \cong D / k(D)$, which is a contradiction. Hence $k(C) \cap k(D)=0$. Now $C \cap k(D)$ is an ideal of $L$ and $C \cap k(D) \subseteq C \cap M$, so $C \cap k(D) \subseteq k(C)$. It follows that $C \cap k(D) \subseteq k(C) \cap k(D)=0$. Similarly $D \cap k(C)=0$.

Next we claim that $(C+k(D)) /(k(C)+k(D))$ is a ideal completion to $M /(k(C)+k(D))$ in $L /(k(C)+k(D))$. For, certainly it is an ideal supplement. Suppose that $X /(k(C)+k(D))$ is an ideal of $L /(k(C)+k(D))$ properly contained in $(C+k(D)) /(k(C)+k(D))$. Then $X \cap C$ is an ideal of $L$ properly contained in $C$, since $X \cap C=C$ implies that $C \subseteq X$ and $X=C+k(D)$. It follows that $X=X \cap(C+k(D))=X \cap C+k(D) \subseteq M$, proving the claim.

Similarly $(D+k(C)) /(k(C)+k(D))$ is an ideal completion to $M /(k(C)+$ $k(D))$ in $L /(k(C)+k(D))$. But now if $k(C)+k(D) \neq 0$ the minimality of 
$L$ implies that $(C+k(D)) /(k(C)+k(D)) \cong(D+k(C)) /(k(C)+k(D))$. But $(C+k(D)) /(k(C)+k(D)) \cong C / k(C)$ and $(D+k(C)) /(k(C)+k(D)) \cong$ $D / k(D)$ so this is a contradiction.

We therefore have $k(C)+k(D)=0$ and $C, D$ are minimal ideals of $L$. Then $L=M+C=M+D$ and $C D \subseteq C \cap D=0$. But now $[M \cap C, L]=$ $[M \cap C, M+D] \subseteq M \cap C$ so $M \cap C$ is an ideal of $L$. It follows that $M \cap C=0$. Similarly $M \cap D=0$. Thus

$$
C \cong \frac{C+D}{D}=\frac{(C+D) \cap(M+D)}{D}=\frac{(C+D) \cap M+D}{D} \cong(C+D) \cap M .
$$

Similarly, $D \cong(C+D) \cap M$, whence $C \cong D$. This contradiction establishes the result.

The ideal index of a maximal subalgebra $M$ in $L, \eta(L: M)$, is the dimension of $C / k(C)$, where $C$ is an ideal completion of $M$ in $L$.

Corollary 2.2 If $M$ is a maximal subalgebra of $L$ then $\eta(L: M)$ is welldefined.

Next we establish how the ideal index behaves with respect to factor algebras.

Proposition 2.3 Let $M$ be a maximal subalgebra of $L$ and let $B$ be an ideal of $L$ with $B \subseteq M$. Let $C / B$ be an ideal completion of $M / B$ in $L / B$, put $k(C / B)=K / B$, and let $D$ be an ideal completion of $M$ in $L$. Then $C / K \cong D / k(D)$.

Proof. In view of Theorem 2.1 we can assume that $D \subseteq C$. We have that $(k(C)+B) / B$ is an ideal of $L$ which is inside $M / B$ and $C / B$, so $k(C) \subseteq K \cap D$. But also $K \cap D$ is an ideal of $L$ and $K \cap D \subseteq M \cap D$ so $K \cap D \subseteq k(C)$. Hence $k(C)=K \cap D$. But now $D \nsubseteq K$ since $K \subseteq M$, so $K+D=C$. This yields that $C / K=(K+D) / K \cong D / D \cap K=\bar{D} / k(D)$.

Corollary 2.4 Let $M$ be a maximal subalgebra of $L$ and let $B$ be an ideal of $L$ with $B \subseteq M$. Then $\eta(L / B: M / B)=\eta(L: M)$. 
Proof. From Proposition 2.3 we have that

$$
\begin{gathered}
\eta(L / B: M / B)=\operatorname{dim}((C / B) / k(C / B))=\operatorname{dim}(C / K) \\
=\operatorname{dim}(D / k(D))=\eta(L: M) .
\end{gathered}
$$

A subalgebra $B$ of a Lie algebra $L$ is called a $c$-ideal of $L$ if there is an ideal $C$ of $L$ such that $L=B+C$ and $B \cap C \leq B_{L}$, where $B_{L}$ is the largest ideal of $L$ contained in $B$. This concept was introduced and studied in [12]. We have the following characterisation of maximal subalgebras that are c-ideals in terms of ideal index.

Theorem 2.5 Let $M$ be a maximal subalgebra of $L$. Then $M$ is a c-ideal of $L$ if and only if $\eta(L: M)=\operatorname{dim}(L / M)$.

Proof. $(\Rightarrow)$ Let $L$ be a Lie algebra of smallest dimension having a maximal subalgebra $M$ which is a c-ideal but for which $\eta(L: M) \neq \operatorname{dim}(L / M)$. If $M_{L} \neq 0$ then $M / M_{L}$ is a c-ideal of $L / M_{L}$, by [12, Lemma 2.1], and so $\eta\left(L / M_{L}: M / M_{L}\right)=\operatorname{dim}\left(\left(L / M_{L}\right) /\left(M / M_{L}\right)\right)$, by the minimality of $L$. But then $\eta(L: M)=\operatorname{dim}(L / M)$, by Corollary 2.4, a contradiction. If $M_{L}=0$, by assumption there is an ideal $C$ of $L$ such that $L=M+C$ and $M \cap C \subseteq M_{L}=0$. Since $M$ is a maximal subalgebra of $L, C$ is a minimal ideal of $L$. But then $\eta(L: M)=\operatorname{dim} C=\operatorname{dim}(L / M)$. This contradiction yields the required implication.

$(\Leftarrow)$ Now let $L$ be a Lie algebra of smallest dimension having a maximal subalgebra $M$ for which $\eta(L: M)=\operatorname{dim}(L / M)$ but $M$ is not a c-ideal of $L$. If $L$ is simple then $\eta(L: M)=\operatorname{dim} L$. But then $M=0$ and $M$ is a c-ideal of $L$, a contradiction. If $M_{L} \neq 0$ then $M / M_{L}$ is a c-ideal of $L / M_{L}$, by the minimality of $L$, giving that $M$ is a c-ideal of $L$, by [12, Lemma 2.1], a contradiction again. Thus we have that $L$ is a non-simple Lie algebra with $M_{L}=0$. Let $B$ be a minimal ideal of $L$. Then $L=M+B$ and $\eta(L: M)=\operatorname{dim} B$. By our assumption then $\operatorname{dim}(L / M)=\operatorname{dim} B$. It follows that $M \cap B=0$ and $M$ is a c-ideal of $L$. This contradiction again establishes the implication.

This yields the following characterisation of solvable Lie algebras in terms of ideal index. 
Corollary 2.6 The Lie algebra $L$ is solvable if and only if $\eta(L: M)=$ $\operatorname{dim}(L / M)$ for all maximal subalgebras $M$ of $L$.

Proof. Simply combine Theorem 2.5 and [12, Theorem 3.1].

With some restrictions on the underlying field the existence of a single solvable maximal subalgebra satisfying the above condition is sufficient to ensure that $L$ is solvable.

Corollary 2.7 Let $L$ be a Lie algebra over a field $F$, where $F$ has characteristic zero or is algebraically closed of characteristic greater than 5. Then $L$ has a solvable maximal subalgebra $M$ with $\eta(L: M)=\operatorname{dim}(L / M)$ if and only if $L$ is solvable.

Proof. Simply combine Theorem 2.5 and [12, Theorems 3.2 and 3.3].

We also have the following characterisation of supersolvable Lie algebras in terms of ideal index.

Corollary 2.8 The Lie algebra $L$ is supersolvable if and only if $\eta(L: M)=$ 1 for all maximal subalgebras $M$ of $L$.

Proof. Suppose first that $L$ is supersolvable and let $M$ be a maximal subalgebra of $L$. Then $L$ has codimension 1 in $L$, by [4, Theorem 7], so $\eta(L: M)=1$ by Corollary 2.6.

Now suppose that $L$ is any Lie algebra and that $M$ is a maximal subalgebra of $L$ with $\eta(L: M)=1$. Then there is an ideal completion $C$ of $L$ with $\operatorname{dim} C / k(C)=1$. Put $C=k(C)+F x$. Then $L=M+F x$ and $M$ has codimension 1 in $L$. But now $L$ is solvable, by Corollary 2.6, and hence supersolvable, by $[4$, Theorem 7$]$.

The Frattini subalgebra of $L, F(L)$, is the intersection of all of the maximal subalgebras of $L$. The Frattini ideal, $\phi(L)$, of $L$ is $F(L)_{L}$.

Theorem 2.9 If $L$ has a supersolvable maximal subalgebra $M$ with $\eta(L$ : $M)=1$ and $N(L) \nsubseteq \mathbb{M}$ then it is supersolvable. 
Proof. Suppose first that $L$ is $\phi$-free. Then $N(L)=A \operatorname{soc}(L)$, by Theorem 7.4 of [10], so there is a minimal abelian ideal $A$ of $L$ with $A \not \nsubseteq M$. Clearly $L=A \oplus M$. Moreover, $\operatorname{dim} A=1$ as in Corollary 2.8 above, and so $L$ is supersolvable.

If $L$ is not $\phi$-free then $\eta(L / \phi(L): M / \phi(L))=1$, by Corollary 2.4, and $N(L / \phi(L))=N(L) / \phi(L)$, by [10, Theorem 6.1], so $L / \phi(L)$ satisfies the hypotheses of the Proposition. It follows from the paragraph above that $L / \phi(L)$ is supersolvable. But then $L$ is supersolvable, by [4, Theorem 6].

We say that the maximal subalgebra $M$ of $L$ has an abelian ideal completion if it has an ideal completion $C$ such that $C / k(C)$ is abelian. Then we have the following result.

Theorem 2.10 The Lie algebra $L$ is solvable if and only if every maximal subalgebra of $L$ has an abelian ideal completion.

Proof. Suppose first that $L$ is solvable and that $M$ is a maximal subalgebra of $L$. Then there exists $k \geq 2$ such that $L^{(k)} \subseteq M$ but $L^{(k-1)} \nsubseteq M$. Put $C=L^{(k-1)}$. Then $L=M+C$ so $C$ is an ideal completion of $M$. Also, $L^{(k)} \subseteq k(C)$, so $C / k(C)$ is abelian.

Suppose now that every maximal subalgebra of $L$ has an abelian ideal completion, let $M$ be a maximal subalgebra of $L$ and let $C$ be an abelian ideal completion of $M$. Then $[C, M \cap C] \subseteq C^{2} \subseteq k(C) \cap C \subseteq M \cap C$, so $M \cap C$ is an ideal of $L$. We infer that $M \cap C \subseteq M_{L}$ and hence that $M$ is a c-ideal of $L$. The result now follows from [12, Theorem 3.1].

Put $F^{*}(L)$ equal to the intersection of all maximal subalgebras of $L$ which have no abelian ideal completion (with $F^{*}(L)=L$ if no such maximal subalgebras exist), and let $\phi^{*}(L)=F^{*}(L)_{L}$. Then we have the following characterisation of the radical of a Lie algebra.

Theorem 2.11 Let $L$ be any Lie algebra. Then $\phi^{*}(L)=R(L)$, the solvable radical of $L$.

Proof. Suppose first that $M$ is a maximal subalgebra of $L$ with $R(L) \nsubseteq M$. Let $C$ be minimal in the set of ideals of $L$ that are inside $R(L)$ but not in $M$. Then $C$ is an ideal completion of $M$ and $C / k(C)$ is a minimal solvable ideal and so abelian. Thus $R(L) \subseteq \phi^{*}(L)$. 
Next suppose that $R(L)=0$. We wish to show that $\phi^{*}(L)=0$. Suppose not, and let $A$ be a minimal ideal of $L$ with $A \subseteq \phi^{*}(L)$. Since $\phi(L)=0$ (see [10, Theorem 6.5]), there is a maximal subalgebra $M$ of $L$ with $A \nsubseteq M$, so $L=M+A$ and $M_{L} \cap A=0$.

Now $F^{*}(L) \nsubseteq M$, so $M$ has an abelian ideal completion $C$. Clearly $L=M+C$ and $M_{L} \cap C=k(C)$. If $A \subseteq C$ then $A^{2} \subseteq C^{2} \cap A \subseteq k(C) \cap A \subseteq$ $M_{L} \cap A=0$ and $A$ is abelian, contradicting the fact that $R(L)=0$. It follows that $A \cap C=0$ whence $[A, C]=0$.

Put $K=\left\{x \in L:[x, C] \subseteq M_{L}\right\}$. Then $M_{L}+C \subseteq K$. Furthermore, $[M \cap K, C] \subseteq[K, C] \subseteq M_{L} \cap K \subseteq M \cap K$, so $M \cap K$ is an ideal of $L$. This yields that $M \cap K \subseteq M_{L}$ from which $K=M \cap K+C \subseteq M_{L}+C$. We therefore have that $K=M_{L}+C$ and $M_{L}+A \subseteq M_{L}+C$. But now

$$
A \cong \frac{A}{M_{L} \cap A} \cong \frac{M_{L}+A}{M_{L}} \subseteq \frac{M_{L}+C}{M_{L}} \cong \frac{C}{M_{L} \cap C}=\frac{C}{k(C)}
$$

and so $A$ is abelian. This is impossible, since $R(L)=0$, so $\phi^{*}(L)=0$.

\section{Maximal completions}

The set $I(M)$ is partially ordered by set inclusion; call maximal elements of $I(M)$ maximal completions of $M$ in $L$. Clearly every ideal completion of $M$ in $L$ is a maximal completion of $M$ in $L$, but the converse is not true in general: for example, if $L$ is the two-dimensional non-abelian Lie algebra with basis $x, y$ in which $[x, y]=y$ and $M=F(x+y)$, then $F x$ is a maximal completion of $M$ in $L$ but is not an ideal of $L$.

Here we are seeking an analogue to a result of Deskins (see [7]): namely, that if a Lie algebra has an abelian maximal completion then it has an abelian ideal completion. As we shall see, this result holds only with conditions on the underlying field. First we consider the structure of Lie algebras with a maximal abelian subalgebra. A Lie algebra $L$ is completely solvable if $L^{(1)}$ is nilpotent.

Proposition 3.1 Let $L$ be a completely solvable Lie algebra. Then $L$ has a maximal subalgebra $M$ that is abelian if and only if either

(i) L has an abelian ideal of codimension one in $L$; or

(ii) $L^{(2)}=\phi(L)=Z(L), L^{(1)} / L^{(2)}$ is a chief factor of $L$, and $L$ splits over $L^{(1)}$. 
Proof. Suppose first that $L$ has a maximal subalgebra $M$ that is abelian. If $M$ is an ideal of $L$ we have case (i). So suppose that $M$ is self-idealising, in which case it is a Cartan subalgebra of $L$. Now $L^{(2)} \subseteq \phi(L) \subseteq M$, by [10, Theorem 6.5]. If $S$ is a subalgebra of $L$ denote by $\bar{S}$ its image under the canonical homomorphism onto $L / L^{(2)}$. Then $\bar{M}$ is a Cartan subalgebra of $\bar{L}$ and $\bar{L}$ has a Fitting decomposition $\bar{L}=\bar{M} \oplus \bar{L}_{1}$. Now $\bar{L}_{1} \subseteq \bar{L}^{(1)}=\overline{L^{(1)}}$ which is abelian, so $\bar{L}_{1}$ is an ideal of $\bar{L}$. Moreover, since $\bar{M}$ is a maximal subalgebra of $\bar{L}, \bar{L}_{1}$ is a minimal ideal of $\bar{L}$ and $\overline{L^{(1)}}=\bar{L}_{1}$. It follows that $L^{(1)} / L^{(2)}$ is a chief factor of $L$. Clearly $\phi(\bar{L})=0$, whence $\phi(L)=L^{(2)}$. Also $L=M+L^{(1)}$, so letting $C$ be a subspace of $M$ such that $M=C \oplus M \cap L^{(1)}$ we see that $C$ is a subalgebra of $L$ and $L$ splits over $L^{(1)}$. Finally, $\left[M, L^{(2)}\right] \subseteq M^{(1)}=0$, so $M \subseteq C_{L}\left(L^{(2)}\right)$. Since $M$ is a self-idealising maximal subalgebra of $L$ and $C_{L}\left(L^{(2)}\right)$ is an ideal of $L$, we have $C_{L}\left(L^{(2)}\right)=L$, whence $L^{(2)}=Z(L)$.

Consider now the converse. If (i) holds the converse is clear. So suppose that (ii) holds. Then $L=C \oplus L^{(1)}$ where $C$ is an abelian subalgebra of $L$. Put $M=C+L^{(2)}$, so $M$ is clearly abelian. Let $M \subseteq T \subseteq L$. Then $\bar{M} \subseteq \bar{T} \subseteq \bar{L}$. But $\bar{L}=\bar{M} \oplus \overline{L^{(1)}}$ and $\overline{L^{(1)}}$ is a minimal abelian ideal of $\bar{L}$. So $\bar{M} \neq \bar{T}$ implies that $\bar{T}=\bar{L}$. It follows that $M$ is a maximal subalgebra of $L$.

Proposition 3.2 Let $L$ be a Lie algebra over an algebraically closed field $F$ of any characteristic. Then $L$ has a maximal subalgebra $M$ that is abelian if and only if either

(i) L has an abelian ideal of codimension one in $L$; or

(ii) $L^{(1)}$ has dimension one and $\phi(L)=0$.

In either case $L$ is completely solvable.

Proof. Suppose first that $L$ has a maximal subalgebra $M$ that is abelian. If $M$ is an ideal of $L$ we have case (i). So suppose that $M$ is self-idealising, in which case it is a Cartan subalgebra of $L$. Let $L=M \oplus L_{1}(M)$ be the Fitting decomposition of $L$ relative to $M$. Then $\left\{\left.(\operatorname{ad} m)\right|_{L_{1}(M)}: m \in M\right\}$ is a set of simultaneously triangulable linear mappings. So, there exists $0 \neq b \in L_{1}(M)$ such that $[m, b]=\alpha(m) b$ for every $m \in M$, where $\alpha(m) \in F$. Then we have that $M+F b$ is a subalgebra of $L$ strictly containing $M$, whence $M \oplus F b=L$. But now $L^{(1)}=F b, \phi(L) \subseteq M \cap L^{(1)}=0$ and we have case (ii).

Consider now the converse. If (i) holds the converse is clear. So suppose that (ii) holds. Then $L=L^{(1)} \oplus M$ for some subalgebra $M$ of $L$, by [10, Lemma 7.2]. Clearly $M$ is an abelian maximal subalgebra of $L$. 
Theorem 3.3 Let L be a Lie algebra over an algebraically closed field and let $M$ be a maximal subalgebra of $L$ with an abelian maximal completion in $L$. Then $M$ has an abelian ideal completion in $L$.

Proof. Let $L$ be a Lie algebra of smallest dimension for which the result is false, and let $M$ be a maximal subalgebra of $L$ with an abelian maximal completion $C$ but no abelian ideal completion. If $L$ is simple then $k(C)=0$ and the maximality of $C$ in $I(M)$ implies that $C=L$, which is impossible.

If $M$ is an ideal of $L$ choose $A$ to be minimal in the set of ideals of $L$ (not necessarily proper) that are not contained in $M$. Then $A$ is an ideal completion of $M$ in $L, A \cap M=k(A)$ and $A / k(A) \cong L / M$ which is one dimensional and hence abelian.

Suppose now that $M_{L}=0$. Then $k(C)=0$ and we can assume that $C$ is not an ideal of $L$. Now $L$ contains a subalgebra $D$ in which $C$ is a maximal subalgebra, and $D$ is solvable, by Proposition 3.2. The result certainly holds if $L$ is solvable, by Theorem 2.10 , so we can assume that $D \neq L$. It follows from the maximality of $C$ in $I(M)$ that $D \notin I(M)$. Since $\langle M, D\rangle=L$ we must have that $k(D) \nsubseteq M$. Thus there is a proper subalgebra $K$ of $D$ such that $K$ is an ideal of $L$ and $L=M+K$. Let $A$ be a minimal non-trivial ideal of $L$ inside $D$. Then $M+A=L, k(A)=0$ and $A$ is abelian because $D$ is solvable. This means that $A$ is an abelian ideal completion of $M$.

Suppose next that $M_{L}=K \neq 0$ and $K \neq k(C)$, and consider the subalgebra $K+C \neq C$. Then $\langle M, K+C\rangle=L$, but $K+C \notin I(M)$ because of the maximality of $C$ in $I(M)$. It follows that $k(K+C) \nsubseteq M$, and so the collection of ideals of $L$ inside $K+C$ but not in $M$ is non-empty. Let $A$ be a minimal element of this set. Clearly $A$ is an ideal completion of $M$ in $L$. Also $(K+C) / K \cong C / C \cap K$ is abelian, since $C / k(C)$ is abelian and $k(C) \subseteq C \cap K$, so $A / k(A)=A / K \cap A \cong(K+A) / K$ is abelian, because $A \subseteq K+C$.

Finally suppose that $M_{L}=K \neq 0$ and $K=k(C)$. If $S$ is a subalgebra of $L$ denote by $\bar{S}$ its image under the canonical homomorphism onto $L / K$. Then $\bar{C}$ is a completion of $\bar{M}$ in $\bar{L}$ and $\bar{M}$ is core-free. If $\bar{C}$ is a maximal element of $I(\bar{M})$, then, by the paragraph above, $\bar{M}$ has an abelian ideal completion $\bar{A}$ in $\bar{L}$. Then $A$ is an ideal completion of $M$ in $L$ and $A / k(A)=$ $A / K \cap A \cong(A+K) / K \cong \bar{A}$ is abelian.

If $\bar{C}$ is not a maximal element of $I(\bar{M})$ then let $\bar{D}$ be minimal amongst those elements of $I(\bar{M})$ which contain $C$ properly. Clearly $C$ is a maximal subalgebra of $D$, so $D$ is not a completion of $M$ in $L$, by the maximality of $C$. Hence $k(D) \nsubseteq \nsubseteq M$. Choose $A$ to be a minimal element in the (nonempty) collection of ideals of $L$ lying in $D$ but not in $M$. Then $A$ is an 
ideal completion of $M$. Also, $D / K$ is solvable, by Proposition 3.2, since $C / K$ is maximal in $L / K$. Moreover, $L / K \supseteq(A+K) / K \cong A / A \cap K$ and $A \cap K=k(A)$, so the chief factor $A / k(A)$ of $L$ is solvable, and thus abelian.

Corollary 3.4 Let L be a Lie algebra over an algebraically closed field. The $L$ is solvable if and only if every maximal subalgebra of $L$ has an abelian maximal completion in $L$.

Proof. This follows from Theorems 2.10 and 3.3.

Note that Proposition 3.2 and Theorem 3.3 do not hold when the underlying field $F$ is not algebraically closed, even if it has characteristic zero, as the following example shows

EXAMPLE 3.1 Let $S$ have basis $e_{1}, e_{2}, e_{3}$ with $\left[e_{1}, e_{2}\right]=-\left[e_{2}, e_{1}\right]=e_{3},\left[e_{2}, e_{3}\right]=$ $-\left[e_{3}, e_{2}\right]=-e_{1},\left[e_{3}, e_{1}\right]=-\left[e_{1}, e_{3}\right]=e_{2}$, all other products being zero (so $L$ is three-dimensional non-split simple), let $\bar{S}$ be an isomorphic copy of $S$ and denote the image of $s \in S$ in $\bar{S}$ by $\bar{s}$. Put $L=S \oplus \bar{S}$ with $[S, \bar{S}]=0$.

Every maximal subalgebra of $S$ is one dimensional, and so abelian, showing that Proposition 3.2 does not hold. Also, it is easy to check that the diagonal subalgebra $M=\{x \in L: x=s+\bar{s}$ for some $s \in S\}$ is maximal in $L$ and that $C=F e_{1}+F \overline{e_{1}}$ is a maximal abelian completion of $M$ in $L$. However, $M$ has no maximal abelian ideal completion in $L$.

\section{Subideal completions}

Let $M$ be a maximal subalgebra of the Lie algebra $L$ and let $S(M)=$ $\{C \in I(M): C$ is a subideal of $L$ and $L=M+C\}$. Clearly, every ideal completion of $M$ in $L$ is in $S(M)$, so $S(M)$ is non-empty. We call elements of $S(M)$ subideal completions of $M$ in $L$.

Then we have the following characterisation of supersolvable Lie algebras in terms of such completions which is analogous to [2, Theorem 1]. To use a similar argument to theirs we would require an underlying field of characteristic zero, as it relies on the Baer radical being a nilpotent ideal (see [1]). The argument given below is independent of the underlying field. 
Theorem 4.1 The Lie algebra $L$ is supersolvable if and only if every maximal subalgebra of $L$ has a subideal completion $C$ with $\operatorname{dim} C / k(C)=1$.

Proof. Suppose first that every maximal subalgebra $M$ of $L$ has a subideal completion $C$ with $\operatorname{dim} C / k(C)=1$. It is clear that $L / \phi(L)$ satisfies the same hypotheses. Moreover, if $L / \phi(L)$ is supersolvable then so is $L$, by [4, Theorem 6], so we can assume that $L$ is $\phi$-free. Since $k(C) \subseteq M$, we have that $M$ has codimension one in $L$. It follows from [11] that $L \cong R \oplus S$, where $R$ is a supersolvable ideal of $L$ and $S$ is a three-dimensional simple ideal of $L$.

Suppose that $S \neq 0$, let $M$ be a maximal subalgebra of $L$ containing $R$, let $C$ be a subideal completion of $M$ with $\operatorname{dim} C / k(C)=1$ and let $\pi: L \rightarrow S$ be the projection homomorphism from $L$ onto $S$. Then $\pi(C)$ is a subideal of $S$, so $\pi(C)=S$ or 0 . Since $\pi(k(C))=0$ the former is impossible. The latter implies that $C \subseteq R$ which is also impossible. It follows that $S=0$ and $L$ is supersolvable.

Conversely, suppose that $L$ is supersolvable. Then $L$ has an ideal (and hence a subideal) completion $C$ with $\operatorname{dim} C / k(C)=1$ by Corollary 2.8 .

\section{References}

[1] R.K. Amayo and I.N. Stewart, Infinite-dimensional Lie algebras, Noordhoof, Leyden (1974).

[2] A. Ballester-Bolinches and Luis M. Ezquerro, On the Deskins index of a maximal subgroup of a finite group, Proc. Amer. Math. Soc. 114 (1992), 325-330.

[3] A. Ballester-Bolinches, Yanming Wang and Guo Xiuyun, Csupplemented subgroups of finite groups, Glasgow Math. J. 42 (2000), 383-389.

[4] D.W. Barnes, On the cohomology of soluble Lie algebras, Math. Z. 101 (1967), 343-349.

[5] J.C. Beidleman and A.E. Spencer, The normal index of maximal subgroups in finite groups, Illinois J. Math. 16 (1972), 95-101.

[6] W.E. Deskins, On maximal subgroups, Proc. Sympos. Pure Math., vol. 1, Amer. Math. Soc., Providence, R.I. (1959), 100-104. 
[7] W.E. Deskins, A note on the index complex of a maximal subgroup, Arch. Math., 54 (1990), 236-240.

[8] N.P. MukherJeE, A note on normal index and maximal subgroups in finite groups, Illinois J. Math. 75 (1975), 173-178.

[9] N.P. Mukherjee and Prabir Bhattacharya, The normal index of a finite group, Pacific J. Math. 132 (1988), 143-149.

[10] D.A. Towers, A Frattini theory for algebras, Proc. London Math. Soc. (3) 27 (1973), 440-462.

[11] D.A. Towers, Lie algebras, all of whose maximal subalgebras have codimension one, Proc. Edin. Math. Soc. 24 (1981), 217-219.

[12] D.A. Towers, C-Ideals of Lie algebras, to appear in Comm. Algebra. 